\title{
Pediatric tetrasomy $18 p$ presenting as a spastic cerebral palsy: A case report
}

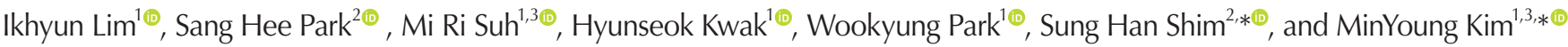 \\ 'Department of Rehabilitation Medicine, CHA Bundang Medical Center, CHA University School of Medicine, Seongnam, Korea \\ ${ }^{2}$ Genetics Laboratory, Fertility Center Gangnam, CHA University, Seoul, Korea \\ ${ }^{3}$ Rehabilitation and Regeneration Research Center, CHA University School of Medicine, Seongnam, Korea
}

\begin{abstract}
Tetrasomy 18p is a genetic syndrome caused by an isochromosome consisting of two copies of the short arm of chromosome 18. Clinically, pediatric cases of tetrasomy 18p manifest with global developmental delay, similar to most cases of chromosomal abnormality. In addition, it causes various symptoms including abnormal muscle tone. We report a case of an infant with global developmental delay and remarkable spasticity, the typical phenotype of bilateral spastic cerebral palsy. However, she had a subtle anomaly in her face, and brain magnetic resonance imaging (MRI) findings were inconsistent with her strong upper motor neuron signs. Upon genetic testing, she was determined to have an 18p isochromosome, confirming de novo non-mosaic tetrasomy 18p. Cerebral palsy is a neurological disorder that includes developmental delay caused by a non-progressive lesion in the developing brain. During diagnostic workup in patients with cerebral palsy, genetic testing should be considered when there are minor physical anomalies or equivocal MRI findings.
\end{abstract}

Key words: Tetrasomy 18p, Cerebral palsy, Hereditary spastic paraplegia.

\section{Introduction}

Tetrasomy $18 p$ or isochromosome $18 p$ (i[18p]) syndrome (Online Mendelian Inheritance in Man \#614290) is caused by an isochromosome, a supernumerary marker chromosome, consisting of two copies of the short arm of chromosome 18. Even though $18 p$ is the most common location of isochromosomes [1], it is still a very rare condition with a prevalence of less than 1 in 40,000 [2]. This syndrome affects both sexs equally, and most are de novo cases [3]. According to a literature review, global de- velopmental delay has been observed in all reported $18 p$ cases. Other features include abnormal muscle tone, seizures, and neonatal feeding problems, which are also frequent symptoms of cerebral palsy $[3,4]$.

Recently, we encountered a pediatric case of de novo nonmosaic tetrasomy $18 p$, clinically diagnosed as spastic cerebral palsy by acquired brain lesion at another hospital. According to a literature review up to 2020, only one such case has been found in Korea, and since this subject had been prenatally diagnosed, their clinical manifestations were not reported [5].

Received: 5 August 2021, Revised: 9 September 2021, Accepted: 10 September 2021, Published: 31 December 2021

${ }^{*}$ Co-corresponding author: Sung Han Shim, Ph.D. iD https://orcid.org/0000-0002-9814-5448

Genetics Laboratory, Fertility Center Gangnam CHA University, 569 Nonhyeon-ro, Gangnam-gu, Seoul 06125, Korea.

Tel: +82-2-3453-6438, Fax: +82-2-3453-6450, E-mail: shshim@cha.ac.kr

MinYoung Kim, M.D., Ph.D. (iD https://orcid.org/0000-0001-5481-2985

Department of Rehabilitation Medicine, CHA Bundang Medical Center, CHA University School of Medicine, 59 Yatap-ro, Bundang-gu, Seongnam 13496, Korea.

Tel: +82-31-780-1872, Fax: +82-31-780-3449, E-mail: kmin@cha.ac.kr

Conflict of interest: The authors declare that they do not have any conflicts of interest.

(c) This is an open-access article distributed under the terms of the Creative Commons Attribution Non-Commercial License (http://creativecommons.org/licenses/by-nc/4.0/) which permits unrestricted non-commercial use, distribution, and reproduction in any medium, provided the original work is properly cited.

(c) Copyright 2021 by the Korean Society of Medical Genetics and Genomics 


\section{Case}

An 8-month-old female visited our department for physical therapy due to delayed motor development. As she was not able to creep forward and had severe spasticity affecting both lower extremities, she was clinically diagnosed with bilateral spastic type of cerebral palsy in another hospital. And acquired brain injury was suspected as the cause of cerebral palsy.

She was born by normal delivery at 41 weeks of gestation with a weight of $2.6 \mathrm{~kg}$ (below 3rd percentile) [6] without any history of trauma or hypoxic events. She was the second child of healthy parents, after her typically developing older sister. At the

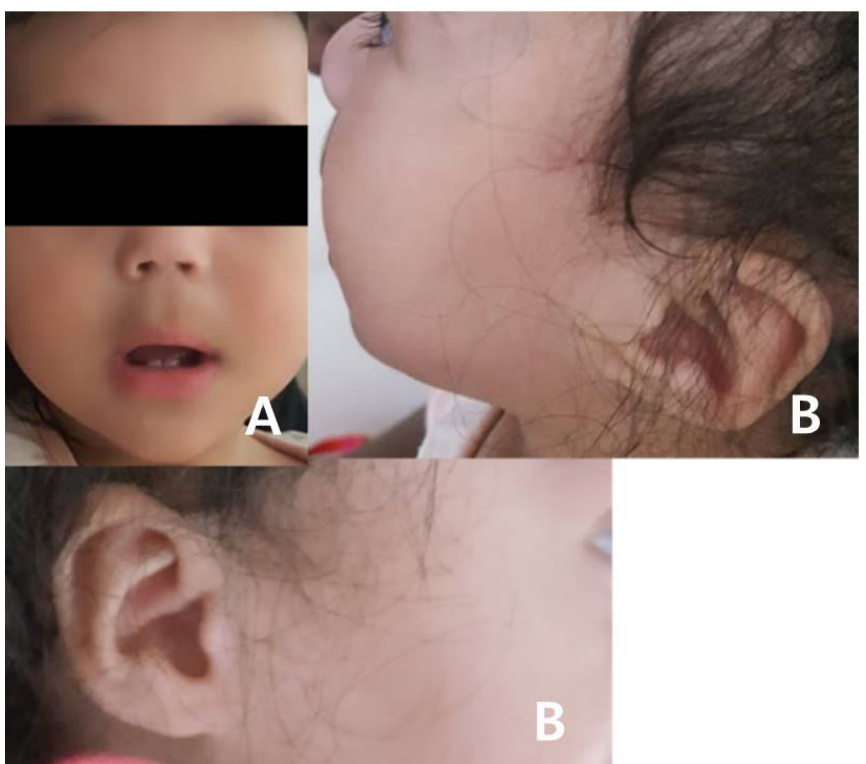

Fig. 1. Dysmorphism observed in the patient. (A) Smooth philtrum. (B) Low set ear. time of birth, her Korean father was 24 years old, and her Malian mother was 26 years old.

At 8 months of age, her body weight was $7.2 \mathrm{~kg}$ (10th-25th percentile), height was $72.6 \mathrm{~cm}$ (90th-97th percentile), and head circumference was $41 \mathrm{~cm}$ (below 5 th percentile, microcephaly). Facial appearance showed low set ear and a smooth philtrum (Fig. 1), which were not found in other family members. Opisthotonic posture and spasticity were observed bilaterally, and we graded her hip adductors as grade 2 , hamstrings as grade $1+$, and heelcords as grade 2 as per the modified Ashworth Scale (MAS). On Bayley Scales of Infant and Toddler Development-2nd edition (BSID-II) evaluation at 9 months [7], her cognitive and psychomotor developmental scores were equivalent to those at 5 months and 4 months of age, respectively.

Brain magnetic resonance imaging (MRI) was performed at 9 months to rule out the possibility of brain lesions, and volume loss of the periventricular white matter and corpus callosum in the parietal area was suspected. Although it mimicked periventricular leukomalacia, the severity of the MRI could not fully explain with clinical severity (Fig. 2).

Electroencephalography showed a few bursts of semirhythmic delta activity on the right side of the head, suggesting focal cerebral dysfunction. Flash visual evoked potential showed delayed latency of P100 waves on both sides. Similar to the gastrointestinal problems in tetrasomy $18 p$ which are reported previously $[3,4]$, she also had severe constipation requiring intermittent lactulose syrup administration. Echocardiography and electrocardiogram screening revealed grossly normal cardiac anatomy with left ventricular hypertrophy. The clinical features of tetrasomy $18 p$ reported in the literature in comparison with the findings in this case are summarized in Table 1.

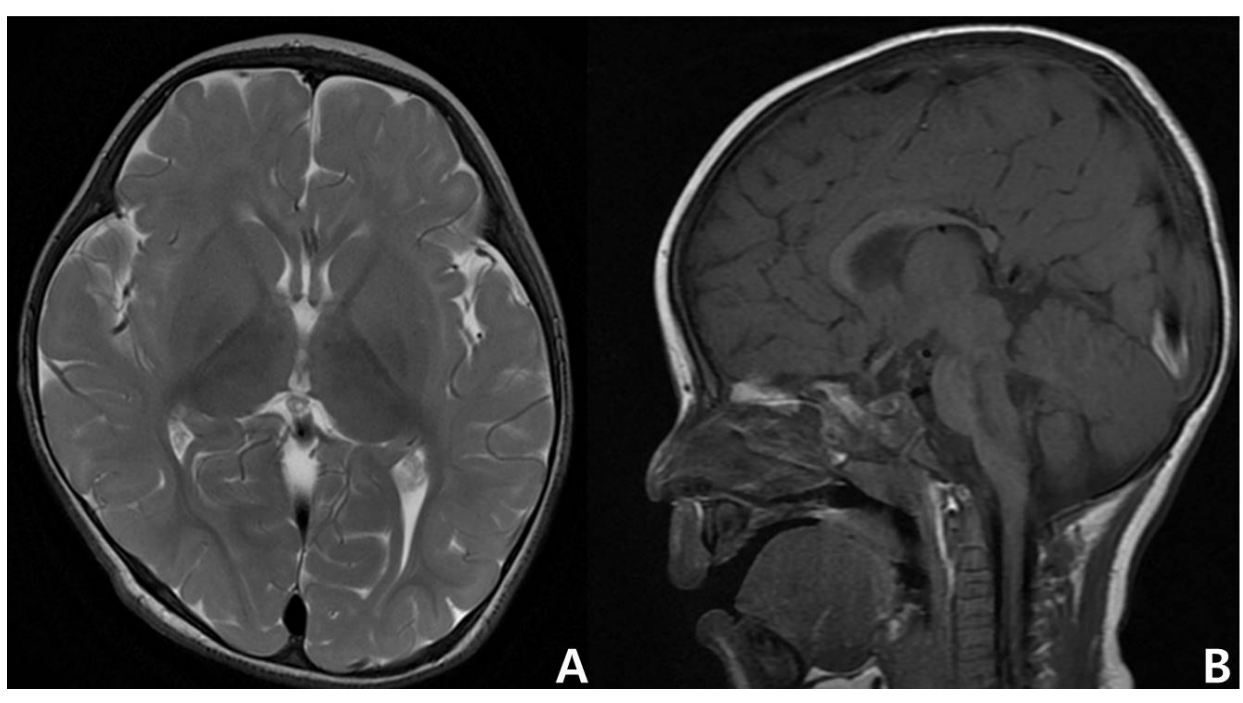

B
Fig. 2. Brain MRI (T1-weighted, axial [A] and sagittal $[\mathrm{B}])$. Suspected decreased white matter volume in parietal areas, with a small splenium and posterior body of corpus callosum. 
Table 1. Comparison of our case with the known phenotype of tetrasomy $18 p$

\begin{tabular}{lccc}
\hline \multicolumn{1}{c}{ Clinical finding } & Literature $^{\text {a }}$ & This case & Total \\
\hline Developmental delay & $108 / 108$ & Yes & $109 / 109$ \\
\hline Dysmorphic features & $108 / 108$ & Yes & $109 / 109$ \\
\hline Smooth philtrum & $27 / 31$ & Yes & $28 / 32$ \\
\hline Small ears & $16 / 31$ & No & $16 / 32$ \\
\hline Abnormal muscle tone & $79 / 108$ & Yes & $80 / 109$ \\
(hypertonia or hypotonia) & & & \\
\hline Congenital heart disease & $23 / 98$ & No & $23 / 99$ \\
\hline Abnormal brain MRI & $11 / 17$ & Yes & $12 / 18$ \\
\hline Myelomeningocele & $6 / 108$ & No & $6 / 109$ \\
\hline Seizure & $23 / 108$ & No & $23 / 109$ \\
\hline Microcephaly & $51 / 108$ & Yes & $52 / 109$ \\
\hline Strabismus & $41 / 90$ & No & $41 / 91$ \\
\hline Recurrent otitis media & $38 / 108$ & No & $38 / 109$ \\
\hline Constipation & $35 / 108$ & Yes & $36 / 109$ \\
\hline Neonatal complication & $70 / 108$ & No & $70 / 109$ \\
\hline Growth retardation & $30 / 108$ & No & $30 / 109$ \\
\hline Hearing loss & $11 / 94$ & No & $11 / 95$ \\
\hline
\end{tabular}

$\mathrm{MRI}$, magnetic resonance imaging.

avalues are presented as data from references [3]/[4].

Data from the article of Sebold C et al. (Am J Med Genet 2010;152:2164-

72) [3]; Bawazeer S et al. (Appl Clin Genet 2018;11:9-14) [4]

\section{Genetic study}

Genetic workup was performed to identify the genetic cause of the dysmorphic features. As the first step, we performed high-resolution chromosome study and multiplex ligation-dependent probe amplification (MLPA) using a subtelomeric probe set (SALSA P070; MRC Holland, Amsterdam, Netherlands) [8], in which a small supernumerary marker chromosome was observed in every metaphase cell. The MLPA results showed a peak ratio of 2.0 at the $18 p 11.32$ region, which strongly suggested that the marker chromosome originated from the short arm of chromosome 18, suggesting tetrasomy 18p (+i[18p]). Fluorescence in situ hybridization (FISH) analysis was conducted using a CEP 18 (D18Z1) SpectrumOrange probe (Abbott Laboratories, Des Plaines, IL, USA) to confirm the diagnosis. On FISH analysis, the supernumerary monocentric marker chromosome showed positive signals (Fig. 3). Consistent with most 18p isochromosome cases being de novo cases, the chromosomal analyses of the parents showed normal karyotypes.

\section{Clinical progress with rehabilitation therapy}

The study patient has received rehabilitation therapy at the
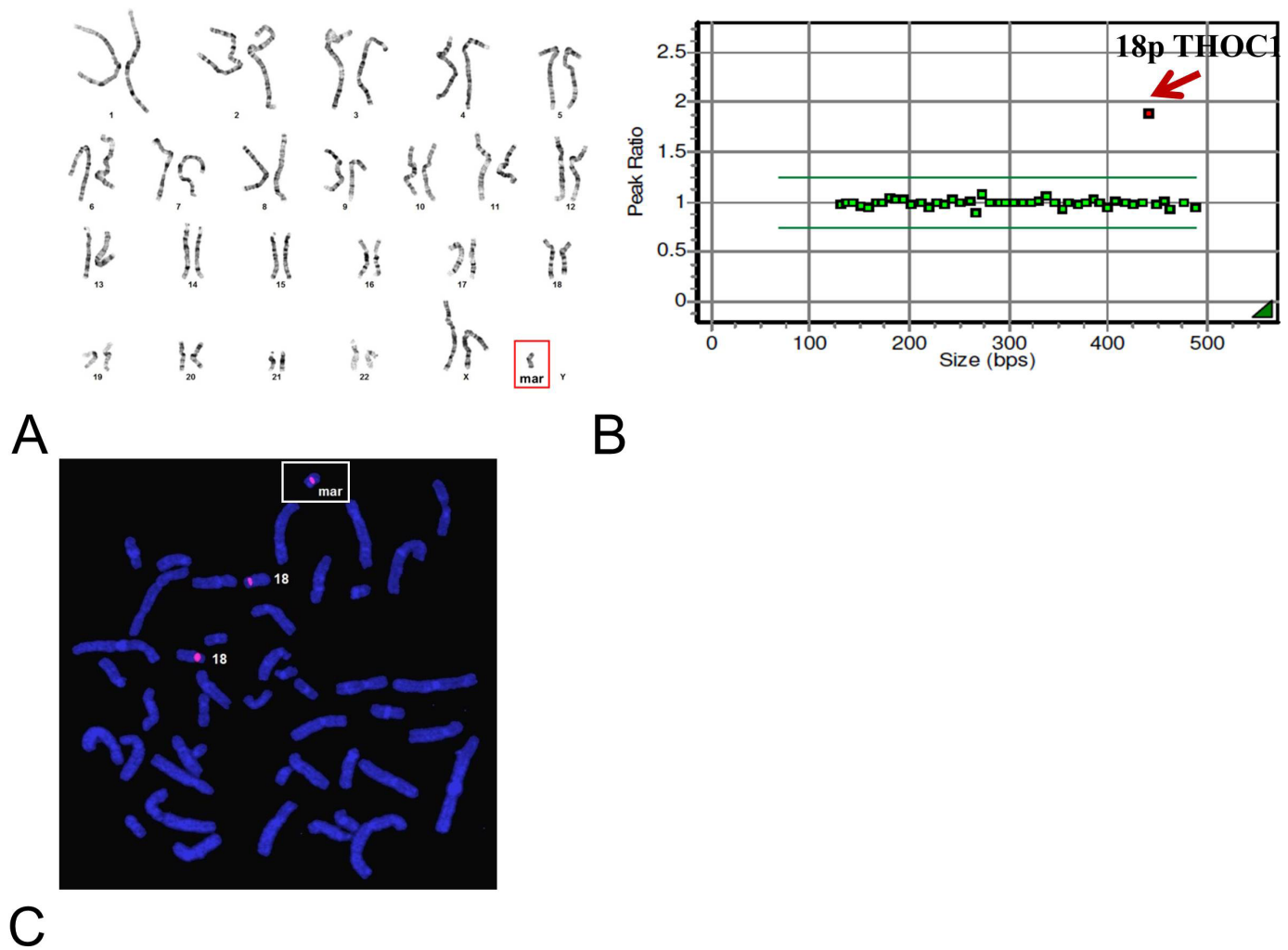

Fig. 3. Genetic testing results. (A) High-resolution chromosome study showed an additional small metacentric marker chromosome. (B) Multiplex ligation-dependent probe amplification results (SALSA P070 subtelomeric probemix) indicated duplication of the 18p11.32 region. (C) Fluorescence in situ hybridization using CEP 18 (D18Z1) Spectrum0range probe showed that monocentric marker chromosome was of chromosome 18 origin. Results summary: 47, XX, +mar.ish i(18)(p10)(D18Z1+). 


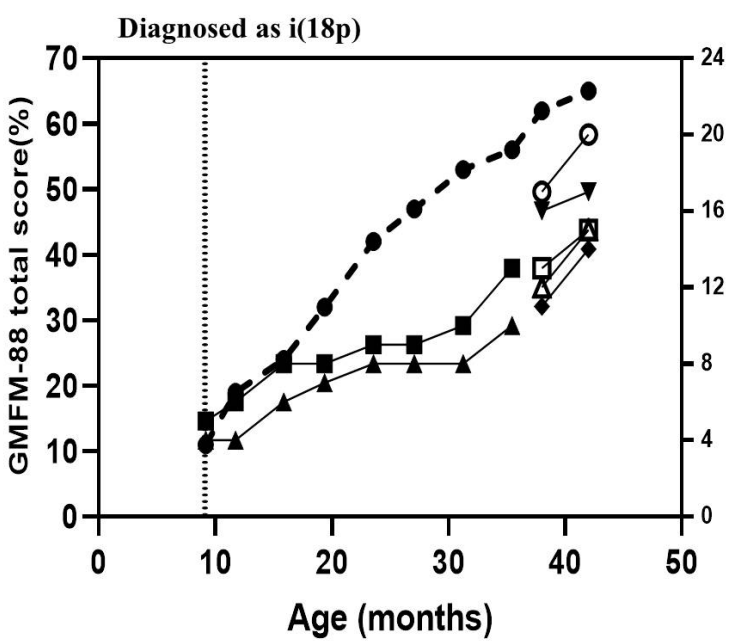

study hospital since her 8 months age via outpatient department and the followings are the latest progresses. At 35 months, she began to crawl forward and stand alone for 3 seconds. From 39 months, she became capable of walking alone more than 10 steps. However, she could not control herself to stand on one leg. Her current (43 months old) functional status is level II on Gross Motor Function Classification System [8]. Her cognitive and gross motor developments were evaluated by BSIDIII, which were equivalent to 17 months and 14 months of age, respectively (Fig. 4). Recently at 45 months, the growth parameters were as follows: height $103.1 \mathrm{~cm}$ (75th to 90th percentile), weight $16.7 \mathrm{~kg}$ (50th to 75th percentile). Constipation much improved, she did not need to take medication. Also, she had no feeding disorders and swallowing difficulties.

Since her hypertonus was considered the biggest problem, we tried antispastic medications. Dantrolene sodium was orally administered at $2 \mathrm{mg} / \mathrm{kg}$ daily from 10 months of age. It was changed to baclofen after 9 months because of non-significant efficacy of dantrolene sodium. Thus, she was orally administered $5 \mathrm{mg}$ of baclofen three times per day for 19 months, when her heel cord spasticity increased to MAS grade 3 , with equinus progression. Due to sufficient amelioration of spasticity, periodic motor point block at both gastrocnemius muscles using botulinum toxin (3 IU/body weight in $\mathrm{kg}$ of onabotulinum toxin $\mathrm{A}$ [Boto ${ }^{\circledR}$, Allergan, Allergan Sales LLC \{Bioscience Laboratories\} Campbell, CA, USA] in each muscle) has been performed since 31 months of age. Spasticity of both heel cords decreased from grade 3 to grade 2 after the first botulinum toxin injection. Additionally, we applied bilateral hinged ankle foot orthoses from 19 months of age to correct equinus gait patterns and facilitate walking. In order to improve gait ability, physical therapy that purposes to enhance balance and lower extremity uses with strengthening exercise has conducted. Occupational therapy purposed to facilitate eye-hand coordination and to stimulate visual perception including puzzle assembly and shape-matching has also provided.

\section{Ethics statement}

The study was approved by the Institutional Review Board of CHA Bundang Medical Center (2020-06-011). The images were published in agreement with the patient's parents.

\section{Discussion}

To our knowledge, this is the first case of tetrasomy $18 p$ in Korea, diagnosed during the workup of developmental delay and showing clinical progress. Previously reported case was discovered during prenatal workup for cardiac defects; thus, clinical courses thereafter was not reported, except for swallowing difficulty [5].

In previous studies, about 63\% of patients showed abnormal brain MRI findings, including thin corpus callosum, brain atrophy, and lateral ventricle enlargement [3]. The study patient had a small periventricular, white matter which has not been reported previously. Although the various neurological features of tetrasomy $18 p$ are seen, brain MRI findings are not frequently reported.

In the present case, the patient's clinical symptoms were very similar to those seen in typical spastic bilateral cerebral palsy caused by periventricular leukomalacia, so she had been undergoing physical therapy in another hospital without further diagnostic workup. However, since the perinatal history and MRI findings could not completely explain the relatively severe clinical features, we conducted a gene study and were able to make 
the appropriate diagnosis.

At the initial stage of diagnostic workup, a genetic condition called hereditary spastic paraplegia (HSP) was considered for diagnosis. HSP is known to be caused by over 80 genetic variants and is often diagnosed through new generation sequencing panel tests [9]. It is not easy to conduct tests in all suspected cases because of high cost. This report suggests that high-resolution chromosomal testing and MLPA may be helpful in some cases presenting with similar symptoms. All isochromosome $18 p$ cases identified to date have been monocentric; the majority are de novo cases, and a few familial cases of maternal and paternal inheritance have been reported $[10,11]$. There are several mechanisms by which chromosome abnormality occur, and meiosis II nondisjunction, followed by subsequent postzygotic misdivsion are the most common mechanisms [10]. In this study, no additional tests such as DNA polymorphism were performed, and the mechanism of isochromosome formation could not be elucidated. Only normal karyotype of the parents revealed that she was a de novo case.

In conclusion, this case implies that we should not overlook the clinical presentation of hypertonicity and be aware of the possibility of genetic causes in pediatric patients with developmental delay presenting with the phenotype of cerebral palsy. Subtle physical anomalies and brain image results may also be important clues for diagnosis.

\section{Acknowledgements}

This research was supported by a grant from the Korea Health Technology R\&D Project through the Korea Health Industry Development Institute (KHIDI), funded by the Ministry of Health \& Welfare, Republic of Korea (grant number: HI16C1559).

\section{Authors' Contributions}

Conception and design: MYK. Acquisition of data: HK, WP, IL. Analysis and interpretation of data: IL, SHP. Drafting the article: IL, SHP. Critical revision of the article: SHS, MRS, MYK. Final approval of the version to be published: all authors.

\section{References}

1. Kotzot D, Bundscherer G, Bernasconi F, Brecevic L, Lurie IW, Basaran $\mathrm{S}$, et al. Isochromosome $18 p$ results from maternal meiosis II nondisjunction. Eur J Hum Genet 1996;4:168-74.

2. O'Donnell L, Soileau BT, Sebold C, Gelfond J, Hale DE, Cody JD. Tetrasomy 18p: report of cognitive and behavioral characteristics. Am J Med Genet A 2015;167:1474-82.

3. Sebold $C_{1}$ Roeder $E_{1}$ Zimmerman $M$, Soileau $B$, Heard $P_{1}$ Carter $E_{1}$ et al. Tetrasomy 18p: report of the molecular and clinical findings of 43 individuals. Am J Med Genet A 2010;152:2164-72.

4. Bawazeer $S$, Alshalan $M$, Alkhaldi A, AlAtwi N, AlBalwi M, Alswaid A, et al. Tetrasomy 18p: case report and review of literature. Appl Clin Genet 2018;11:9-14.

5. Jung PS, Won HS, Cho IJ, Hyun MK, Shim JY, Lee PR, et al. A case report of prenatally diagnosed tetrasomy 18p. Obstet Gynecol Sci 2013;56:190-3.

6. Kim JH, Yun S, Hwang SS, Shim JO, Chae HW, Lee YJ, et al.; Committee for the Development of Growth Standards for Korean Children and Adolescents; Committee for School Health and Public Health Statistics, the Korean Pediatric Society; Division of Health and Nutrition Survey, Korea Centers for Disease Control and Prevention. The 2017 Korean National Growth Charts for children and adolescents: development, improvement, and prospects. Korean J Pediatr 2018;61:13549.

7. Bayley N. Bayley scales of infant development. 2nd ed. New York: Psychological Corporation, 1993.

8. Wu Y, Ji T, Wang J, Xiao J, Wang H, Li J, et al. Submicroscopic subtelomeric aberrations in Chinese patients with unexplained developmental delay/mental retardation. BMC Med Genet 2010;11:72.

9. Parodi L, Coarelli G, Stevanin G, Brice A, Durr A. Hereditary ataxias and paraparesias: clinical and genetic update. Curr Opin Neurol 2018;31:462-71.

10. Bugge $M$, Blennow $E_{1}$ Friedrich $U$, Petersen MB, Pedeutour $F_{1}$ Tsezou $A$, et al. Tetrasomy 18p de novo: parental origin and different mechanisms of formation. Eur J Hum Genet 1996;4:160-7.

11. Eggermann T, Engels $H_{1}$ Apacik $C$, Moskalonek B, Müller-Navia J, Schwanitz G, et al. Tetrasomy $18 p$ caused by paternal meiotic nondisjunction. Eur J Hum Genet 1997;5:175-7. 